\title{
Die Richtungsadverbien in den deutschen Dialekten Mährens und Schlesiens
}

\section{Mojmír Muzikant}

The article deals with the dialectal forms of selected directional adverbs and their territorial distribution in the former German dialects in Moravia and Silesia. The selection is limited to adverbs which express movement 'inside, upwards and over something', namely in the direction to and from the speaker. Closer examination shows that, on average, each adverb appears in 13 to 15 different dialectal variants which can be further differentiated according to frequency and location. Some variants occur only sporadically without forming a spatially connected areal. Other variants are more frequent and typical for certain areas; therefore, they can be considered as specific features of the respective dialect. For the examined dialectal area as a whole, it is typical that there is hardly any homogeneity with respect to the occurrence of the examined direction adverbs. The highest degree of diversity can be found in a certain area of the so called Schönhengst region. On the other hand, equalizing tendencies can be observed in large cities and in another area of the Schönhengst region as well as in the eastern part of northern Moravia.

Directional adverbs; dialectal variants; territorial distribution; German dialects in Moravia and Silesia

Im vorliegenden Artikel wird die mundartliche Wiedergabe von ausgewählten Richtungsadverbien und ihre territoriale Verbreitung untersucht. Die Auswahl ist auf solche Formen beschränkt, die die Richtung nach innen, nach oben und über etwas angeben, und zwar je nachdem, ob die Bewegung auf den Standpunkt des Sprechers zu oder vom Standpunkt des Sprechers weg erfolgt. Es zeigt sich, dass jedes von den behandelten Adverbien im Schnitt 13 bis 15 Varianten aufweist, wobei diese aus quantitativer und räumlicher Sicht weiter differenziert werden können. Einige Varianten sind nur vereinzelt und ohne territorialen Zusammenhang belegt. Einige treten dagegen in größerer Zahl auf und sind nur für bestimmte Teilgebiete charakteristisch, sodass man in diesen Fällen von spezifischen Merkmalen der entsprechenden Dialekte sprechen kann. Für den untersuchten Dialektraum ist typisch, dass in den Teilgebieten in Bezug auf die Richtungsadverbien keine Homogenität herrscht. Die größte Formenvielfalt weist der Schönhengst auf. In Großstädten sowie in einigen weiteren Ortschaften des Schönhengsts und des östlichen Teils Nordmährens sind Nivellierungstendenzen zu beobachten.

Richtungsadverbien; mundartliche Wiedergaben; territoriale Verbreitung; deutsche Dialekte in Mähren und Schlesien

\section{Einleitung}

Die Tschechoslowakei hatte um 1930 ca. 14,5 Mio. Einwohner, davon waren etwa 3,2 Mio. deutschstämmig. Weil die deutschen Kolonisten größtenteils unbesiedeltes Gebiet eingenommen hatten, bildeten sich in Böhmen und Mähren, besonders an der historischen Grenze, kompakte deutschsprachige Territorien heraus, die einmal 
einen zunächst breiteren, dann wieder schmaleren Streifen rings um ganz Böhmen und Mähren bildeten (vgl. KMS 2011:7). In der Nachkriegszeit verringerte sich der Anteil der deutschsprachigen Bevölkerung infolge der Aussiedelung (Vertreibung) drastisch. Bei der Volkszählung im Jahre 2001 bezeichneten sich in der ganzen Republik nurmehr etwas über 38000 Menschen als „Deutsche“ (vgl. Bachmann 2005:73).

Das Ziel des vorliegenden Beitrags ist es, die Formenvielfalt der Richtungsadverbien in den deutschen Dialekten Mährens und Schlesiens zu erfassen. Der Frage nach der territorialen Zugehörigkeit der erhobenen Formen zu Dialektgebieten im deutschen Kernland soll dabei an dieser Stelle nicht mehr systematisch nachgegangen werden, da diese Problematik bereits an anderem Ort (s. Bachmann 2005; Muzikant 2013; Muzikant 2014) ausreichend behandelt worden ist. (Knappe Bemerkungen zu diesem Punkt finden sich aber verstreut in den jeweiligen Kapiteln zu den einzelnen Richtungsadverbien sowie im Fazit des Beitrags.) Der Untersuchung lag die Erwartung zugrunde, dass sich aus der Menge der untersuchten Formen bestimmte Regularitäten für die territoriale Verbreitung im untersuchten Dialektraum ergeben könnten. Auf der einen Seite sollten sich Belege identifizieren lassen, die in unterschiedlichen Teilgebieten auftreten (allgemeine Merkmale), auf der anderen Seite dagegen Belege, die nur für ein bestimmtes Teilgebiet kennzeichnend sind (spezifische Merkmale).

\section{Zur Herkunft des verwendeten Sprachmaterials}

Als Quelle für die Beschreibung und Auswertung der mundartlichen Wiedergaben von Richtungsadverbien wurden die Fragebücher herangezogen, die im Rahmen des Projekts „Sprachatlas der deutschen Dialekte in Mähren und Schlesien“ im Zeitraum 2001-2008 erstellt wurden. Dem Belegkorpus in Form von ausgefüllten Fragebüchern liegen Erhebungen aus insgesamt 84 Ortschaften zugrunde, die vom Autor des vorliegenden Beitrags und seinen Mitarbeitern Richard Rothenhagen, Marek Halo und Šárka Pugnerová durchgeführt worden sind.

Die Gewährspersonen (GP) waren zur Zeit der Erhebungsphase zwischen 70 bis 90 Jahre alt und lebten überwiegend noch auf dem Gebiet der Tschechischen Republik. Als eine ideale Gewährsperson war ein Dialektsprecher anzusehen, der im bäuerlichen Milieu aufgewachsen ist und an seinem Geburtsort lebt. Nur die Nichtausgesiedelten hatten u. E. die besten Voraussetzungen dafür, ihren einheimischen Dialekt zu bewahren, weil sie nicht den Einflüssen der deutschen Standardsprache sowie der dialektalen Umgebung in der neuen Heimat ausgesetzt waren. Bei der Suche nach geeigneten GP waren jedoch Kompromisse zu machen. Es hat sich herausgestellt, dass in manchen Gegenden weiße Flecken entstehen, weil gute Dialektsprecher bereits verstorben waren. Aus diesem Grund sahen wir uns gezwungen, uns nach geeigneten Dialektsprechern im Ausland umzusehen. 
Dies war vor allem bei der Untersuchung in der Brünner SI sowie in Nordmähren (Hotzenplotzer Gebiet/ Osoblažsko) der Fall. Insgesamt wurden im Ausland fünf Erhebungen durchgeführt, wobei vier davon auf Sprecher, die ursprünglich in der Brünner SI gelebt haben, entfallen. Im Einzelnen sind dies folgende Erhebungen: die Mödritzer Mundart in Wolkersdorf bei Wien, die Schöllschitzer Mundart in Wien, die Maxdorfer Mundart in Sindelfingen (Deutschland), die Morbeser Mundart in Sandhausen bei Heidelberg (Deutschland) und die Hotzenplotzer Mundart in Hof (Deutschland).

\section{Herein}

Die dominierende Variante für das Richtungsadverb herein ist die Form rai/räi, die im Kernland im Ostfränkischen zu Hause ist (vgl. KBS 2006: 98 f.). Sie ist mit Ausnahme der Wischauer SI in allen Teilgebieten des untersuchten Dialektraums belegt. In der Brünner SI, in der SI Wachtl/Deutsch Brodek, ferner im Schönhengst und in Nordmähren ist sie vorherrschend: $g$ Hi $m r a$ Schöllschitz, g Ho bn $r$ a 9 Deutsch Brodek, gHum $r$ a A 5 Dittersdorf, g Ho h $r$ a A 5 Pföhlwies, g Hum r a 5 Liebenthal. In der Iglauer und Olmützer SI ist diese Variante in der Minderheit: g Hu m r a A 5 Iglau, g Hu $m$ r a 9Schnobolin. Die im Schönhengst (Trübauer Gebiet) zweimal verzeichnete Form $r \bar{a}$ kann als Ergebnis einer Monophthongierung erklärt werden: g Ha H $r$ a OPirkelsdorf, g Hu $m$ r a $\theta$ Langenlutsch. Im südöstlichen Teil des Schönhengsts (Trübauer Gebiet und das Gebiet Dörfles-Kornitz) ist z. T. die Form roi/rōi zu verzeichnen, die im Kernland für das Rheinfränkische (das Gebiet um Aschaffenburg) typisch ist (vgl. KBS 2006: 98): g Hu m r O 5Mariendorf, g Hu m r O B 5 Hinter-Ehrnsdorf, g Hu m r 0 A 5 Kornitz. Verhältnismäßig häufig ist die Form rain mit Wegfall des Erstgliedes und $n$-Erhaltung im Auslaut. Vereinzelt steht sie in der Iglauer, Brünner und Olmützer SI: g Hum ra A G Simmersdorf, g Ho nh ra i Wojkowitz, g Hum ra Neustift. Eine größere Belegdichte dieser Variante ist im Schönhengst sowie in Nordmähren (Hultschiner Ländchen, Troppauer, Sternberger

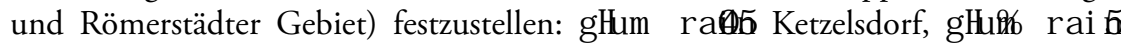

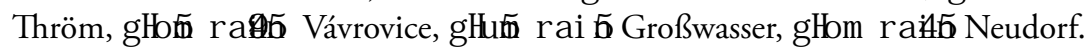

Für die Iglauer SI ist die Form āi $\operatorname{az}$ kennzeichnend, die durch Schwund des anlautenden Konsonanten zu erklären ist: g Hum a 5 Blumendorf, g Hum a A 5 Wolframs. Nasalierte Formen beweisen, dass im Anlaut ursprünglich ein Nasal vorhanden gewesen war: $g$ Hum $m 5$ Stannern, g Hum 5 Neustift bei Iglau, g Hum a A 8 Schrittenz. Die in der Wischauer SI belegten Formen ənī, enez gehen auf das Adverb einher mit stark reduzierter Endung zurück, das nach KBS (2006: 99) im Kernland für die östliche Hälfte Bayerns (Oberpfalz, Nieder-, Oberbayern) typisch

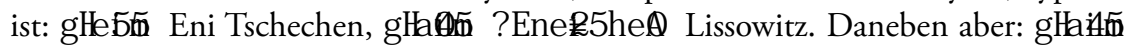
Er i n Hobitschau. 
In Ortschaften mit Nivellierungstendenzen erscheinen die Richtungsadverbien, wie in der Standardsprache, mit beiden Wortbildungskomponenten: $\mathrm{g} \mathrm{Ho} \mathrm{H}$

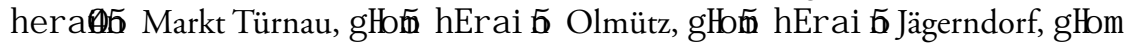
her vom Sprecher weg zum Ausdruck: ge $\theta$ hi naA Brünn. Die gegensätzliche Richtung wird auch durch die Form mit Kürzung der anlautenden Silbe nai-signalisiert: g i 0 n a 5 Stangendorf, gi na A 5Pobutsch, g i PA ( n a 5 Hof. Mehrmals ist die Verwendung des Richtungsadverbs her zu verzeichnen (Schönhengst, Nordmähren): g Hu $m$ ha Putzendorf, g Hu $\mathrm{m}$ ha $\theta$ Unter-Langendorf, g Ho th hal Groitsch.

\section{Hinein}

Ähnlich wie bei herein wird im Fall des Richtungsadverbs hinein das Erstglied der präfigierten Form stark gekürzt. Fast alle Teilgebiete weisen verhältnismäßig häufig die Variante nai/nāi auf. Sie ist lediglich in der Wischauer und Olmützer SI nicht vertreten. In der Iglauer SI kommt sie vereinzelt vor: $g$ e \% I a 5 ë 5 na 5 Blumendorf, gi ogobds I a $\mathrm{A}$ \& na 4 4Maxdorf, gi Ozdi 5 nae Wachtl,gi ogänds

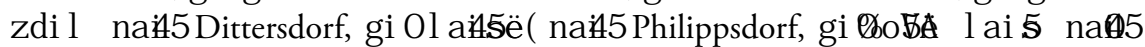
Kunewald. Im Trübauer Gebiet des Schönhengsts ist außerdem die Variante nōi sowie die monophthongierte Form $n \bar{a}$ zu verzeichnen: gi 0 OBA gänds zdil noE5 Mariendorf, gi $00 B A$ Zdi 5 noB 5 Hinter-Ehrnsdorf, gi 0 OB zdi 5 na $\theta$ Langenlutsch. Eine unbedeutende Rolle spielt die Form nain, die sporadisch im Schönhengst, in der Olmützer SI sowie in Nordmähren (Odergebirge, Sternberger Gebiet) zu finden ist: gi 0 gänds I OAs na Ketzelsdorf, geogänds I a A ë 5 naA naA E Domstadtl.

In der Iglauer SI erscheint mehrmals wie im Fall von herein die Form äi mit Schwund des anlautenden Konsonanten: ge 051 a 5 ë ? a 5 Stannern, ge $05 \mathrm{~g}$ änds z dü 0 a 5 Neustift bei Iglau, ge $\mathbf{5}$ z di 5 a 5 Schrittenz. Die in der Wischauer SI vorkommenden Formen gehen auf das Adverb einhin zurück, das nach KBS (2006: 99) im Kernland für die östliche Hälfte Bayerns kennzeichnend ist: ge 55

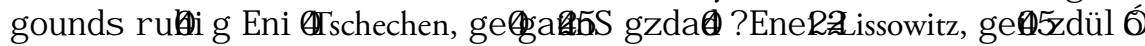
EnEi $\overline{~ H o b i t s c h a u . ~ R i c h t u n g s a d v e r b i e n ~ m i t ~ b e i d e n ~ W o r t b i l d u n g s k o m p o n e n t e n ~}$ hinain, hināi treten sporadisch im Schönhengst sowie in Nordmähren (v. a. in großen Städten) auf: gi $0 \mathrm{a}$ gänds I a 5 hi na 5 Unter-Heinzendorf, ge 0 a A

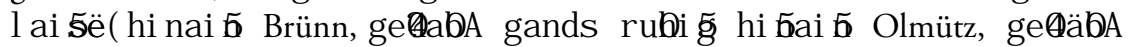

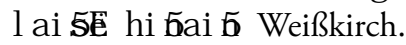

Häufig belegt sind in unserem Material verkürzte Formen, die von her in der Bedeutung ,hin“ wie etwa in der norddeutschen Umgangssprache gebildet werden (vgl. Duden 1998: 366). Sie kommen in den Varianten rai, rain, vereinzelt $r \bar{a}$ vor. 
Sie sind in der Iglauer, Brünner und Olmützer SI, ferner im Schönhengst und in Nordmähren in ziemlich großer Zahl festzustellen: ge $\mathrm{B} 5 \mathrm{l}$ a $\mathrm{s} \mathrm{r}$ a Wolframs, ge $\theta$ go \%6 ds I a A 5 ë $5 r$ a 5 Mödritz, ge $\theta z d i$ \% r i 5 Schönbrunn, ge $\theta$ ? o be $5 \mathrm{~g}$ ands

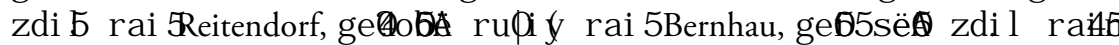
Simmersdorf, ge日gänds I a

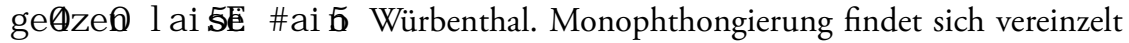
im Trübauer Gebiet des Schönhengsts: $g e \theta g a ̈ n d s ~ z d i l$ ra $\&$ Putzendorf. Das Richtungsadverb herein ist in der Brünner SI einmal belegt: $g$ e Oa A z di 5 he $\bar{B}$ a A $\mathrm{a}$ Wojkowitz.

\section{Herauf}

Die häufigste Wiedergabe für herauf ist die verkürzte Form rauf. Sie ist in der Brünner SI und in der SI Wachtl/Deutsch Brodek dominierend: sogi $\mathrm{m}$ ? eE suldsE ? ins rao4 ghe日an Priesenitz, sëg na a 4 sëb raovghuma Wachtl. Sehr häufig kommt raufim Schönhengst vor: $s$ äg i an do5a4ra $4 v$ dsu ? uns g Hil mo Landskron, sog i on el sël dsu mi A rauvghuma Kornitz. In

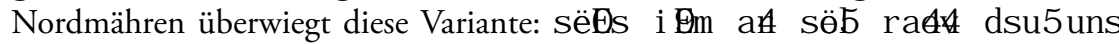
g Ho mA Goldenstein, sëOnen da eE dsu uns \#a $\theta$ gHume 5säE Römerstadt,

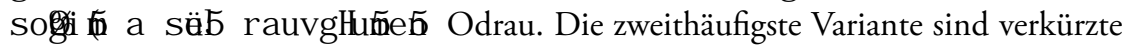
Formen mit kurzem Monophthong im Zweitglied ruf, rof, raf. In Nordmähren konzentrieren sich diese Formen auf den nordwestlichen Zipfel, das Gesenke und

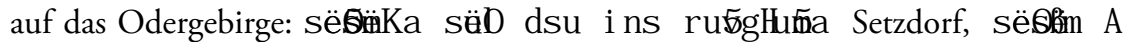
sël Ruvghom Philippsdorf, sëHo z sind sie auch im Hotzenplotzer Gebiet, an der westlichen Grenze Nordmährens, im Freudenthaler Gebiet sowie im östlichen Teil Nordmährens zu finden: $s \ddot{\theta}$ ต dasadso ? ús rugghi and Hotzenplotz, sepog a su\% dso ?ubs rub.

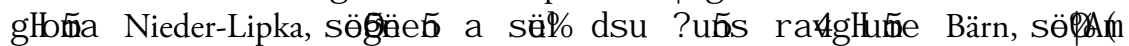
a s äБ2 r u g g Hu mA Sedlnitz. Im Schönhengst kommt vereinzelt die Form ruf vor: sog i on dos a 4 r uvg Huma $4 \mathrm{~s} \ddot{4} \%$ Tirpes. Für das Trübauer Gebiet sowie den Ostschönhengst ist die Form mit langem $\bar{a}$ im Zweitglied (das Erstglied her-wird nicht realisiert) kennzeichnend: $50 \mathrm{gAn}$ e së $\mathrm{r}$ rag Huma $4 \mathrm{dsu}$ ? uns HinterEhrnsdorf, sog a A En el sërraghuma Langenlutsch, sägnEn eE sël dsu 5 u $\overline{\text { G } \mathrm{r}} \mathrm{r} \mathrm{g} \mathrm{g}$ Ho $\mathrm{mA}$ Kirchles. Darüber hinaus ist diese Form isoliert in der Iglauer SI

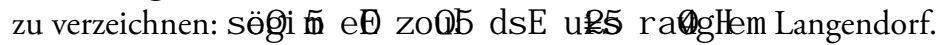

Auf das Richtungsadverb aufher mit stark gekürztem Zweitglied, das für die östliche Hälfte Bayerns kennzeichnend ist (vgl. KBS 2006: 99), gehen die in der

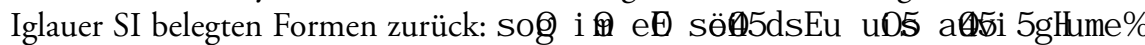

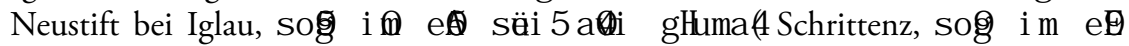

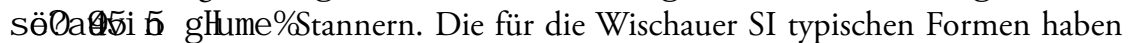
in anderen Teilgebieten des untersuchten Dialektraums keine Analogie: $s$ äg i the A 


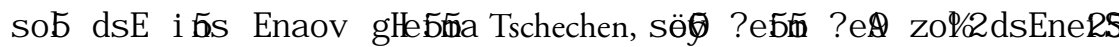

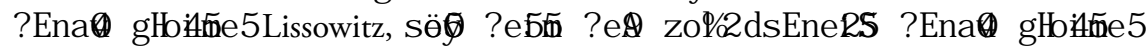
Hobitschau.

Die übrigen Varianten kommen selten vor. Im Zwittauer Gebiet des Schönhengsts

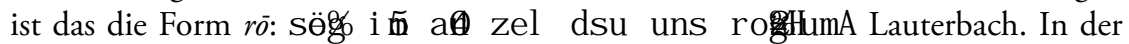
Olmützer SI und im Römerstädter Gebiet Nordmährens handelt es sich um die monophthongischen Formen naf, nuf: $50 \mathrm{~g}$ i on dos el së $\mathrm{d}$ ds u uns na g Hu ma 4 Nimlau, sëlöé a säi Gnug ghume 5Klein Mohrau. Die diphthongische Form nauf tritt mehrmals im Schönhengst und in Nordmähren auf: 50 ge 6 a $4 z$ e $\mathrm{O}$ e 5 uns

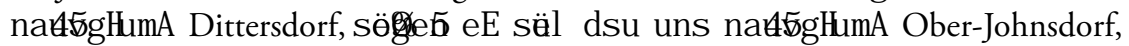

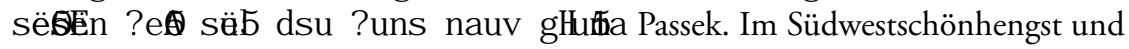
im Zwittauer Gebiet des Schönhengsts ist die zweite Komponente nauf mit Wegfall des auslautenden Spiranten belegt: süOg dsu dag a zeb5dsu ús nebio g Hu ma 4Mährisch Rothmühl, s 09 e a z z eds E uns no log Hu mA Deutsch Biela, sole bs a A z i Onogohuma Stangendorf.

Richtungsadverbien mit beiden Wortbildungskomponenten sind erwartungsgemäß in Ortschaften mit Nivellierungstendenzen (v. a. in großen

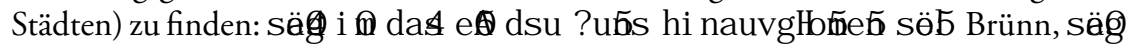

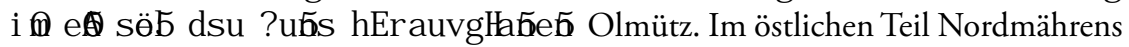
und in der nördlichen Hälfte der Iglauer SI ist die Umschreibung in die Höhe belegt: sü日 dA säl dsu5uns a Al i Gdhe Blumendorf. Im Hultschiner Ländchen (Nordmähren) findet sich der Ausdruck nach oben:sag i on ? e sob I i

\section{Hinauf}

Die häufigste Variante ist die verkürzte Form nauf, die in der Brünner SI, in der SI Wachtl/Deutsch Brodek, ferner im Schönhengst und in Nordmähren vorherrschend ist: geaznel nats Mödritz,gioznel nate Deutsch Brodek, gi Oznel natb Ober-Johnsdorf, geazneb naov Augezd, geoznel na 6 b. Neudorf, ge0zneb nauv Großwasser. Darüber hinaus ist nauf noch in der Olmützer SI vertreten: $g$ e $\theta$ z nel $n a \theta *$ Schnobolin. Keine Belege für diese Variante gibt es in der Iglauer und Wischauer SI. In Nordmähren spielen die monophthongischen Formen nuf, nof eine bedeutende Rolle. Sie sind besonders im nordwestlichen Zipfel und im Gesenke verbreitet:gi0? z zneb nub Buchsdorf,ge日zneb nub Würbenthal. Darüber hinaus sind sie auch im Römerstädter und Freudenthaler Gebiet, im Odergebirge und im östlichen Teil Nordmährens zu finden: gi |e z ne 5 nub Klein Mohrau, ge4 znel no4. Spachendorf, geb5 znel no4. Alt-Lublitz, gi 0 zne\% nub Sedlnitz. Im Trübauer Gebiet des Schönhengsts sowie im Ostschönhengst tritt die Variante näf auf: gi $\theta$ z ne $\bar{b}$ na 0 Hinter-Ehrnsdorf. In zwei Ortschaften des Zwittauer Gebiets und des Südwestschönhengsts ist eine monophthongische Form 
mit Wegfall des auslautenden Spiranten (nō) belegt: g i 0 z ne 5 n o ob Stangendorf, ge znöl \{no OMährisch Rothmühl.

Von Belang sind in unserem Material auch verkürzte Richtungsadverbien, deren reduziertes Erstglied $(r-)$ auf die deiktische Komponente her zurückgeführt werden könnte. Den Formen würde dann eine Verwechslung von her und hin zugrunde liegen, die in den verkürzten Formen in den hier untersuchten Mundarten nicht selten vorkommt (s. o. nauv, nuf, nof für herauf). In diphthongischer Gestalt (rauf) sind solche Formen vor allem im Schönhengst und in der Iglauer SI, vereinzelt auch in Nordmähren anzutreffen: g e $\theta z$ n e $b$ r a u v Brüsau, g e $\theta z$ ne $b$ r a u v Schönbrunn, ge日 znöi $5 r a \notin v$ Iglau, ge日zneb rauv Mährisch Aussee. Monophthongische Formen mit kurzem Vokal (ruf, rof, raf) konzentrieren sich auf das Odergebirge: $g$ e $\theta$ z nel ra 4. Bodenstadt, ge a z nel rov. Klein-Glockersdorf. Monophthongische Formen mit langem Stammvokal $(r \bar{o}, r \bar{o} f, r \bar{a} f)$ finden sich in sehr beschränkter Zahl

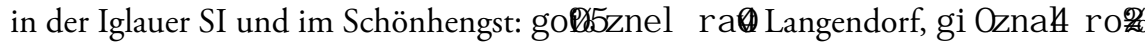
Lauterbach, gi 0 gezVind $r$ or Kirchles.

In der Iglauer SI überwiegen sonst in der östlichen Hälfte Bayerns verwendete Adverbien, die auf aufhin zurückgehen (vgl. KBS 2006:98 f.): ge B $5 \mathrm{~g}$ z Vi nd ? a 8 i i Stannern, geb5gZVind a $\theta$ vi Wolframs, ge日ls gzVind a i Simmersdorf. Für die Wischauer SI gilt eine umgekehrte Folge der Wortbildungskomponenten des Adverbs - deiktische Komponente, relationale Komponente: g e A $5 \mathrm{~g}$ Z V i Ad E r a o V Tschechen, ge日znöb2? Ena0 Lissowitz, ge日5g Vind Enabs Hobitschau.

Der Standardsprache nahestehende Richtungsadverbien mit ungekürzten Komponenten finden sich vereinzelt in der Brünner und Olmützer SI sowie im

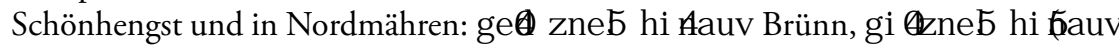
Olmütz, getznel hi nat4. Budigsdorf, getzneb hi bauv Messendorf, gei 5 z nel hi na\& Groß-Olbersdorf. Umschreibungen durch in die Höhe, nach oben kommen selten vor. Die Präpositionalfügung in die Höhe ist in der Iglauer SI und im östlichen Teil Nordmährens zu finden: ge \% g z Vi nd i $6 \mathrm{dhe}=5$ Blumendorf,

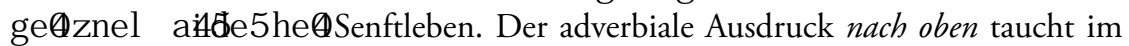
Hultschiner Ländchen sowie im Sternberger und Freudenthaler Gebiet auf: $g$ e $\theta z$ n e 5

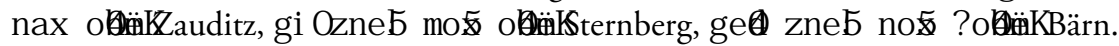

\section{Herüber}

Am häufigsten sind gekürzte Formen mit dem stark reduzierten Erstglied her belegt. Der entrundete $i$-Vokal wird dabei in mehreren Fällen (in Nordmähren) zu é gesenkt. Vorherrschend ist diese Variante in der Brünner SI, in der SI Wachtl/ Deutsch Brodek und in Nordmähren: $r$ i $\theta$ Mödritz, r i $\theta$ e 5Wachtl, g Hu $m$ r i De Buchsdorf, $r$ e Reihwiesen. Sehr verbreitet ist diese Form im Schönhengst: r i OA Schönbrunn, r i BA Lichwe. In Senftleben (östlicher Teil Nordmährens) wird der bilabiale Plosiv im Inlaut zum labiodentalen Aproximanten: $r$ e $r$ Senftleben. 
Im Hultschiner Ländchen sowie in einigen weiteren Ortschaften (Römerstädter, Troppauer Gebiet, östlicher Teil Nordmährens) bleibt der gerundete $\bar{u}$-Vokal

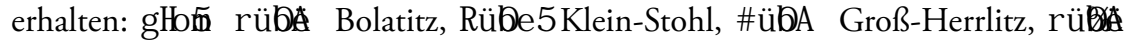
Odrau. Sporadisch ist diese Erscheinung auch im Ostschönhengst zu verzeichnen: g Ho 的 $r$ ü̈A Hohenstadt, \# ̈̈BA Augezd.

Eine große Formenvielfalt weist der Schönhengst auf. Neben den o. g. Formen kommen dort vor allem solche Adverbien vor, bei denen die Diphthonge $u i$, ai, $o i$ variieren. Es zeigt sich dabei eine gewisse territoriale Verteilung. Im Zwittauer Gebiet sind es Richtungsadverbien mit $u i$ im Inlaut, in einem Fall mit der ungekürzten Komponente her verbunden: rui br Lauterbach, g Hum r ui bAt $p$ Hopfendorf, r ui BA Stangendorf, hErui BA Deutsch Biela. Für das Trübauer Gebiet sind Formen mit inlautendem ai typisch, in mehreren Fällen mit ungekürzter Komponente her: gHum $\mathrm{r}$ aABA Hinter-Ehrnsdorf, $\mathrm{r}$ a A BA Langenlutsch, gHi d s u $5 r$ a 5 A Putzendorf, her a A b ë 5Pohler, he r a 5 \&m Mariendorf. Der Diphthong $o i$ ist in einem Fall im Ostschönhengst belegt: he r o \% A Kirchles. Darüber hinaus sind im Schönhengst in beschränktem Maße standardsprachliche Formen sowie das Richtungsadverb her für , herüber' anzutreffen: he r i OA Mährisch Trübau, he r i BA Unter-Heinzendorf, $\mathrm{h}$ a A Laubendorf, he $\mathrm{O}$ ha QKetzelsdorf, g Ho m ha Schönwald.

Die Formen her, hierher für ,herüber' sind in geringem Umfang auch in anderen Teilgebieten (Iglauer SI, Wischauer SI, Nordmähren) bekannt: he Stannern, g Ha A f n he A Hobitschau, g Hom he Vávrovice. Die der Standardsprache nahestehenden Richtungsadverbien sind im Großen und Ganzen verhältnismäßig häufig. Außerhalb des Schönhengsts sind sie vereinzelt in der Iglauer SI, häufiger dann in der Wischauer und Olmützer SI sowie in Nordmähren zu finden: he r i 0 A Neustift

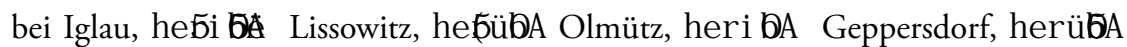
Groß-Olbersdorf. Eine Besonderheit Nordmährens sind Formen mit ì im Inlaut. Sie kommen hauptsächlich im Gesenke und im östlichen Teil Nordmährens vor:

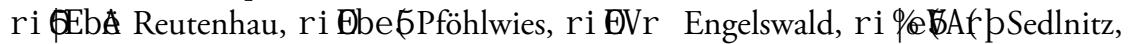
g Hum r i Q⿴囗十 K Kunewald. Für die Iglauer SI und z. T. auch für die Olmützer SI ist der Gebrauch der Präposition über in der Bedeutung, hinüber' spezifisch: I OA Simmersdorf, i DAn Wolframs, i De 5Nimlau. Als isolierte Formen sind folgende Belege zu betrachten: hr i 0 L Langendorf, hi B Bm Schnobolin.

\section{Hinüber}

Ähnlich wie bei dem Gegenpol herüber ist die häufigste Variante die Form mit stark reduziertem Erstglied, in diesem Fall also hin. Der entrundete $\bar{i}$-Vokal im Zweitglied - ̈̈ber wird in Nordmähren mehrmals zu è gesenkt. Vorherrschend ist diese Variante in der Brünner SI und in der SI Wachtl/Deutsch Brodek sowie in Nordmähren: duAd ni OA Schöllschitz, ni Be 5 Wachtl, ni Bë Setzdorf, ni BA Großwasser, ne A Reihwiesen, ne A Altstadt. Recht häufig ist diese Form im Schönhengst: ge On i B A 
Kornitz, gi 0 n i OA Landskron. Vereinzelt ist sie in der Wischauer und Olmützer SI belegt: ni Ö̀ 5 Hobitschau, ni OA Schnobolin. In Senftleben (östlicher Teil Nordmährens) wird der bilabiale Plosiv im Inlaut zum labiodentalen Aproximanten: $n$ e $r$ Senftleben. Die Rundung bleibt in einigen wenigen Fällen im Schönhengst und in Nordmähren erhalten: nüBA Mährisch Rothmühl, g e Qn ü B⿺A Messendorf.

Im Schönhengst kommen Formen mit Diphthongen im Inlaut vor. Für das Zwittauer Gebiet ist der Diphthong $u i$ typisch, in einigen Fällen mit der deiktischen Komponente hin verbunden: nuibr Lauterbach, gi 0 nui bAtp Hopfendorf, hEnui BA Deutsch Biela. Im Trübauer Gebiet hat das Richtungsadverb hinüber nicht selten den Diphthong ai im Inlaut: gi 0 na A 6 A Hinter-Ehrnsdorf, na A BA Langenlutsch, hi na A BA Mariendorf. Im Ostschönhengst tritt der Diphthong oi auf. Er ist nur in einer Ortschaft belegt: h i no \% \% A Kirchles.

Verkürzte Formen mit der deiktischen Komponente her, z. T. mit entrundetem Vokal im Inlaut, finden sich in geringer Anzahl in der Brünner SI und im Schönhengst: r üBA Wojkowitz, ri B Priesenitz, ge日 r übA Hohenstadt, ri BA Zwittau. In Nordmähren sind sie hauptsächlich im Hultschiner Ländchen anzutreffen: ge $\theta$ r ̈̈日 Zauditz, ge日 r ü日 Bolatitz. Daneben: $r$ i $\theta$ ë5Reitendorf, re日 UnterLangendorf, r i $\quad$ Tschimischl. Standardnahe Formen mit beiden ungekürzten Komponenten sind mit Ausnahme der Iglauer SI und der SI Wachtl/Deutsch Brodek in allen Teilgebieten nur in beschränktem Maß vertreten: hi nü日A Brünn,

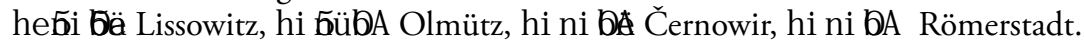

Eine Sonderstellung nimmt die Iglauer SI ein. Die dort vorkommenden Belege sind z. T. auf die ostbairische Form überhin (vgl. KBS 2006: 99) zurückzuführen: i Oeri g Stannern, i Deri 5 Neustift bei Iglau, i OErE Wolframs. Darüber hinaus sind dort einige isolierte Formen zu finden, die in anderen Teilgebieten nicht vorkommen: $d r i \theta A d$ Blumendorf, $h r i b$ Langendorf. In einigen Fällen (in der Iglauer und Wischauer SI sowie im Schönhengst) erscheint anstelle des Richtungsadverbs die Präposition über in scheinbar selbständiger Stellung. Bei genauerem Hinsehen ist ersichtlich, dass die Gewährsleute einen konkreten Ort als anschließendes Substantiv angeführt haben: ge 05 i $\theta$ A de $\operatorname{E}$ Ve Schrittenz, ge 5 i BAn bos Iglau, i AA, po Tschechen, geO i de Vel Triebendorf, gi 0 i 0 A di go5 Pobutsch. Ausschließlich in Ketzelsdorf (Zwittauer Gebiet des Schönhengsts) ist die folgende Form belegt: $d r$ r U B ह̈ Б Ketzelsdorf.

\section{Ergebnisse}

Zusammenfassend lässt sich Folgendes sagen: Im Hinblick auf die vorgestellten mundartlichen Wiedergaben für die untersuchten Richtungsadverbien fallen bestimmte territoriale Besonderheiten in der Verbreitung der einzelnen Varianten auf. Einige Varianten kommen ausschließlich in bestimmten Teilgebieten vor. Man kann sie in dieser Hinsicht als spezifische Merkmale für die jeweiligen Regionen ansehen. Dies ist der Fall bei den Richtungsadverbien herein, hinein, herauf, hinauf in 
der ISI als (a i /a D, a f $\mathrm{i}$ /a uf $\mathrm{f}$ i ) und in der WSI als (en i benei n, ena 0 /enauf ). Durch spezifische Merkmale, die sonst nirgendwo auftauchen, zeichnet sich auch

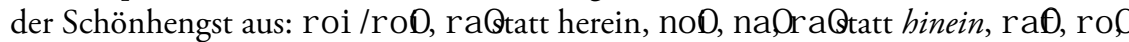
no Ostatt herauf und na 0 , hi na 0 , no ostatt hinauf. Im Gegensatz zu WSI und ISI befinden sich dort die obengenannten Merkmale in Symbiose mit Varianten, die häufig v. a. in Nordmähren zu verzeichnen sind. Aus der Sicht der Formenvielfalt nimmt der Schönhengst somit den ersten Platz ein.

Die meisten Varianten, die in Nordmähren belegt sind, sind (bis auf die WSI und die ISI) mehr oder weniger in allen anderen Teilgebieten verbreitet, was ihre Einordnung als allgemeine Merkmale berechtigt. Eine Ausnahme stellen die Formen ruflrofl raffür herauf und nuflnoffür hinauf bzw. Ri $\mathrm{\theta be}$, $\mathrm{n}$ i $\mathrm{\theta b}$ b für herüber, hinüber dar, die als spezifische Merkmale von Nordmähren und Schlesien aufzufassen sind.

Es ist interessant, dass bei den Richtungsadverbien herüber und hinüber die ISI und die WSI ihre Sonderstellung als Bastion von Dialektmerkmalen, die sonst für die östliche Hälfte Bayerns typisch sind (vgl. KBS 2006: 101), eingebüßt haben, weil hier solche Formen verwendet werden, die auch in anderen Teilgebieten Mährens üblich sind. Die der Standardsprache nahestehenden Richtungsadverbien finden sich v. a. in Großstädten wie Brünn und Olmütz sowie im Hultschiner Ländchen. Darüber hinaus gibt es einige weitere Ortschaften mit starken Nivellierungstendenzen wie Markt Türnau im Trübauer Gebiet des Schönhengsts und Daub im östlichen Teil Nordmährens.

Die oben angeführte Aufzählung von unterschiedlichen Richtungsadverbien weist auf eine bunte Vielfalt von Sprachmitteln in den verschiedenen Existenzformen der Sprache hin und könnte somit den DaF-Lehrern und -Lehrerinnen einen Eindruck davon vermitteln, dass es neben den Formen, die man in der Schule gelernt hat, noch andere geben kann, die von deutschen Muttersprachlern verwendet werden. Vielleicht könnte diese Einsicht dazu beitragen, im Unterricht weniger rigoros mit den Äußerungen von Lernern umzugehen und auch solche Ausdrücke, die nicht in normierten Nachschlagewerken zu finden sind, nicht unbedingt gleich als „falsch“ zu bezeichnen.

\section{Literatur}

Bachmann, Armin R. (2005): Nordbairisches in den deutschen Mundarten Tschechiens. In: Kanz, Ulrich / Wildfeuer, Alfred (Hgg.): Kreuther Kräuterbuschen. Beiträge zur 9. Bayerisch-österreichischen Dialektologentagung in Wildbad Kreuth September 2004. Regensburg 2005, edition vulpes. S. 73-83.

DUDEN, Grammatik der deutschen Gegenwartssprache. 6., neubearb. Aufl. Mannheim [u. a.], Dudenverlag/Bibliographisches Institut, 1998.

Muzikant, Mojmír (2013): Herkunftsgebiete mundartlicher Elemente Nordmährens. In: Strömungen in der Entwicklung der Dialekte und ihrer Erforschung. Beiträge zur 11. Bayerisch-österreichischen Dialektologentagung in Passau September 2010. Regensburg 2013, edition vulpes. S. 343-355. 
Muzikant, Mojmír (2014): Personalpronomen im Schönhengst als mögliches Indiz für die Herkunft der deutschen Siedler. In: Linguistica Pragensia 2/2014. S. 120-135.

Muzikant, Mojmír/Rothenhagen, Richard (2011): Kleiner Mährischer Sprachatlas der deutschen Dialekte. Brno, Masaryk-Universität.

Renn, Manfred/König, Werner (2006): Kleiner Bayerischer Sprachatlas. München, Deutscher Taschenbuchverlag.

\section{Abkürzungen}

BSI Brünner Sprachinsel KMS Kleiner Mährischer Sprachatlas

GP Gewährspersonen SCH Schönhengst

ISI Iglauer Sprachinsel SI Sprachinsel

KBS Kleiner Bayerischer Sprachatlas WSI Wischauer Sprachinsel

\section{Quellen}

Fragebücher zur Erstellung des Sprachatlasses der deutschen Mundarten in der Tschechischen Republik

\section{Quellennachweis der Abbildungen}

Abb. 1: Eigens vom Autor für den Beitrag erstellte Abbildung.

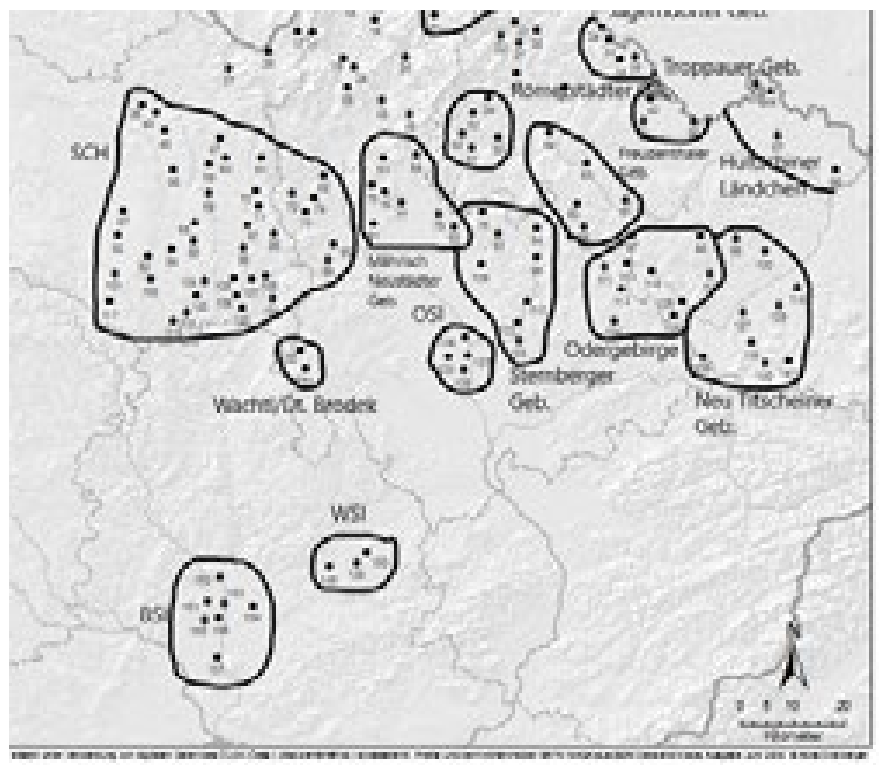

Abb. 1: Untersuchungsgebiete Mährens und Schlesiens 


\section{Liste der erhobenen Ortschaften}

01 Rosenkranz/Růženec

02 Ober-Gostitz/ Horní Hoštice

03 Waldek/Zálesí

04 Jauernig/ Javorník

05 Buchsdorf/Buková

06 Stachlowitz/Stachlovice

07 Neuhäuser/Nové Chaloupky

08 Niesnersberg/Nýznerov

09 Setzdorf/Vápenná

10 Neu-Rothwasser/Nová Červená Voda

11 Urlich/Javořina

12 Freiwaldau/Jeseník

13 Hollunder/Chebzí

14 Reihwiesen/Rejvíz

15 Obergrund/Horní Údolí

16 Johannesthal/Janov

17 Groß-Mohrau/Velká Morava

18 Kunzendorf/Kunčice

19 Goldenstein/Branná

20 Adamsthal/Adamov

21 Philipsdorf/Filipovice

22 Buchbergsthal/Železná

23 Einsiedel/Mnichov

24 Geppersdorf/Linhartovy

25 Hotzenplotz/Osoblaha

26 Nieder-Lipka/Dolní Lipka

27 Glasdörfl /Sklené

28 Hannsdorf/Hanušovice

Heinzendorf an der March/ Hynčice nad Moravou

30 Reutenhau/Rejhotice

31 Würbenthal/Vrbno pod Pradědem

32 Adamsthal/Adamov

33 Schönwiese/Krásné Loučky

34 Weißkirch/Kostelec u Krnova

35 Jägerndorf/Krnov

36 Lichwe/Libchavy

37 Linsdorf/Těchonín

38 Pföhlwies/Lužná

39 Klein Mohrau/Malá Morávka
79 Deutsch Hause/Huzová

80 Hof/Dvorce

81 Alt-Lublitz/Staré Lublice

82 Hopfendorf/Chmelík

83 Stangendorf/Vendolí

84 Zwittau/Svitavy

85 Ketzelsdorf/Koclírov

86 Mährisch Trübau/Moravská Třebová

87 Altstadt/Staré Město

88 Pirkelsdorf/Prklišov

89 Lechowitz/Lechovice

90 Augezd/Újezd

91 Mährisch Aussee/Úsov

92 Passek/Paseka

93 Dohle/Dalov

94 Bärn/Moravský Beroun

95 Domstadtl/Domašov nad Bystřicí

96 Gundersdorf/Guntramovice

97 Tschirm/Čermná ve Slezsku

98 Groitsch/Gručovice

99 Altstadt/Stará Ves

100 Groß-Olbersdorf/Velké Albrechtice

101 Laubendorf/Pomezí

102 Mährisch Rothmühl/Radiměr

103 Ober-Heinzendorf/Horní Hynčina

104 Pohler/Pohledy

105 Langenlutsch/Dlouhá Loučka

106 Mariendorf/Mařín

107 Putzendorf/Pacov

108 Markt Türnau/Městečko Trnávka

109 Sternberg/Šternberk

110 Großwasser/Hrubá Voda

111 Rudelzau/Rudoltovice

112 Liebenthal/Luboměr pod Strážnou

113 Bernhau/Barnov

114 Klein-Glockersdorf/Klokočůvek

115 Fulnek/Fulnek

116 Engelswald/Mošnov

117 Schönbrunn/Jedlová 
Engelsberg/Andělská Hora

Kunau/Kunov

Braunsdorf/Brumovice

Thröm/Třebom

Zauditz/Sudice

Černowir/Černovír

Knappendorf/Knapovec

Ober-Johnsdorf/Horní Třešňovec

Reitendorf/Rapotín

Rudelsdorf/Rudoltice

Altendorf/Stará Ves

Römerstadt/Rýmařov

Neudorf/Nová Ves

Klein-Stohl/Malá Štáhle

Messendorf/Mezina

Groß-Herrlitz/Velké Heraltice

Vávrovice/Vávrovice

Bolatitz/Bolatice

Ribnik/Rybník

Rudelsdorf/Rudoltice

Landskron/Lanškroun

Schönwald/Strážná

Hohenstadt/Zábřeh

Rabersdorf/Hrabišín

Tschimischl/Třemešek

Spachendorf/Leskovec nad Moravicí

Ludgersthal/Ludgeřovice

Lauterbach/Čistá

Dittersdorf/Dětřichov

Tirpes/Trpík

Budigsdorf/Krasíkov

Triebendorf/Třebařov

Unter-Heinzendorf/Dolní Hynčina

Kirchles/Krchleby

Pobutsch/Pobučí

Rohle/Rohle

Steine/Kamenná

Markersdorf/Nová Hradečná

Unter-Langendorf/Dlouhá Loučka

Mojmír Muzikant

Pedagogická fakulta MU
118 Deutsch Biela/Bělá nad Svitavou

119 Brüsau/Březová nad Svitavou

120 Hinter-Ehrnsdorf/Zadní Arnoštov

121 Kornitz/Chornice

122 Posluchau/Posluchov

123 Nirklowitz/Mrsklesy

124 Bodenstadt/Potštát

125 Wessiedel/Veselí

126 Odrau/Odry

127 Kunewald/Kunín

128 Sedlnitz/Sedlnice

129 Blumendorf/Květnov

130 Pattersdorf/Bartoušov

131 Langendorf/Dlouhá Ves

132 Wachtl/Skřípov

133 Deutsch Brodek/Brodek u Konice

134 Schnobolin/Slavonín

135 Nimlau/Nemilany

136 Olmütz/Olomouc

137 Neustift /Nové Sady

138 Daub/Dub

139 Neu-Titschein/Nový Jičín

140 Seitendorf/Životice u Nového Jičína

141 Senfleben/Ženklava

142 Simmersdorf/Smrčná

143 Schrittenz/Strrítež

144 Wolframs/Kostelec

145 Neustift bei Iglau/Cerekvička

146 Iglau/Jihlava

147 Stannern/Stonařov

148 Tschechen/Čechyně

149 Lissowitz/Lysovice

150 Hobitschau/Hlubočany

151 Morbes/Moravany

152 Brünn/Brno

153 Priesenitz/Př́zřenice

154 Maxdorf/Dvorska

155 Schöllschitz/Želešice

156 Mödritz/Modřice

157 Wojkowitz/Vojkovice

Katedra německého jazyka a literatury (a. D.)

E-Mail: mojmir.muzikant@seznam.cz 